This article was downloaded by: [Indest open Consortium]

On: 17 July 2009

Access details: Access Details: [subscription number 907749878]

Publisher Taylor \& Francis

Informa Ltd Registered in England and Wales Registered Number: 1072954 Registered office: Mortimer House, 37-41 Mortimer Street, London W1T 3JH, UK

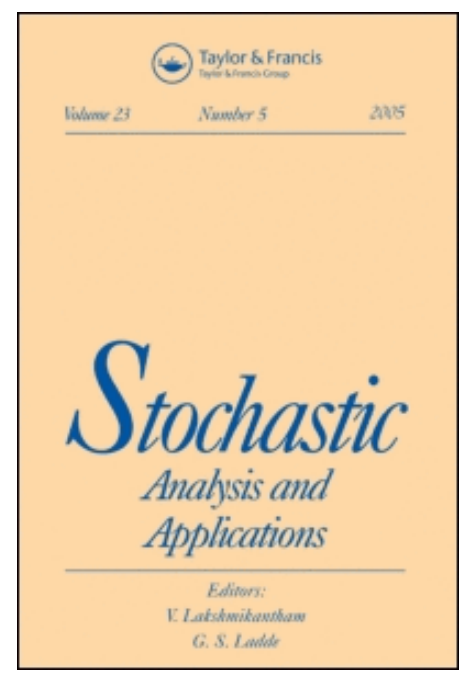

\title{
Stochastic Analysis and Applications
}

Publication details, including instructions for authors and subscription information:

http://www.informaworld.com/smpp/title content=t713597300

\section{Ergodic control of degenerate diffusions}

Gopal K. Basak a; Vevek S. Borkar b; Mrinal K. Ghosh ${ }^{\mathrm{b}}$

a Dept.of Mathematics, Hong Kong University of Science and Technology, Clear Water Bay, Kowloon, Hong

Kong ${ }^{b}$ Dept. of Electrical Engineering, Indian Institute of Science, Bangalore, India

Online Publication Date: 01 January 1997

To cite this Article Basak, Gopal K., Borkar, Vevek S. and Ghosh, Mrinal K.(1997)'Ergodic control of degenerate diffusions',Stochastic Analysis and Applications, $15: 1,1-17$

To link to this Article: DOI: $10.1080 / 07362999708809460$

URL: http://dx.doi.org/10.1080/07362999708809460

\section{PLEASE SCROLL DOWN FOR ARTICLE}

\footnotetext{
Full terms and conditions of use: http://www.informaworld.com/terms-and-conditions-of-access.pdf

This article may be used for research, teaching and private study purposes. Any substantial or systematic reproduction, re-distribution, re-selling, loan or sub-licensing, systematic supply or distribution in any form to anyone is expressly forbidden.

The publisher does not give any warranty express or implied or make any representation that the contents will be complete or accurate or up to date. The accuracy of any instructions, formulae and drug doses should be independently verified with primary sources. The publisher shall not be liable for any loss, actions, claims, proceedings, demand or costs or damages whatsoever or howsoever caused arising directly or indirectly in connection with or arising out of the use of this material.
} 


\title{
ERGODIC CONTROL OF DEGENERATE DIFFUSIONS
}

\author{
Gopal K. Basak * \\ Dept. of Mathematics \\ Hong Kong University of Science and Technology \\ Clear Water Bay, Kowloon, Hong Kong \\ mabasak@uxmail.ust.hk \\ Vivek S. Borkar ${ }^{\dagger}$ \\ Dept. of Electrical Engineering \\ Indian Institute of Science \\ Bangalore 560 012, India \\ vborkar@vidyut.ee.iisc.ernet.in \\ Mrinal K. Ghosh \\ Dept. of Mathematics \\ Indian Institute of Science \\ Bangalore 560 012, India \\ mrinal@math.iisc.ernet.in
}

\begin{abstract}
We study the ergodic control problem of degenerate diffusions on $\mathbb{R}^{d}$. Under a certain Liapunov type stability condition we establish the existence of an optimal control. We then study the correponding HJB equation and establish the existence of a unique viscosity solution in a certain class.
\end{abstract}

\footnotetext{
-Research partially supported by grant no. D4087 DAG 93/94 SC29

${ }^{t}$ Research supported by grant no. 26/01/92-G from DAE, Govt. of India
}

Copyright $\mathbb{O} 1997$ by Marcel Dekker, Inc. 


\section{INTRODUCTION}

We address the problem of controlling degenerate diffusions by continually monitoring the drift. The objective is to minimize the long-run average (ergodic) cost over all admissible controls. The state $X(t)$ of the system at time $t$ is governed by

$$
d X(t)=b(X(t), u(t)) d t+\sigma(X(t)) d W(t), t \geq 0, \quad X(0)=X_{0}
$$

where $b: \mathbb{R}^{d} \times U \rightarrow \mathbb{R}^{d}$ and $\sigma: \mathbb{R}^{d} \rightarrow \mathbb{R}^{d \times d}$ are respectively the drift vector and the diffusion matrix, $U$ is a given compact metric space which is the control set. $X_{0}$ is a prescribed $\mathbb{R}^{d}$-valued random variable and $W(\cdot)$ is a $d$-dimensional standard Wiener process independent of $X_{0} \cdot u(\cdot)$ is a non-anticipative control process taking values in $U$. Such a control is called an admissible control. Our aim is to minimize over all admissible controls the following quantity :

$$
\limsup _{T \rightarrow \infty} \frac{1}{T} E \int_{0}^{T} c(X(t), u(t)) d t
$$

where $c$ is the running cost function. If the minimum is obtained for some $u(\cdot)$, it is called an optimal control. Under certain conditions we will show that there exists an optimal control. We then study the corresponding Hamilton-Jacobi-Bellman (HJB for short) equation. The HJB equation for this problem is given by

$\inf _{u}\left[\frac{1}{2} \sum_{i, j, k=1}^{d} \sigma_{i k}(x) \sigma_{j k}(x) \frac{\partial^{2} \phi(x)}{\partial x_{i} \partial x_{j}}+\sum_{i=1}^{d} b_{i}(x, u) \frac{\partial \phi(x)}{\partial x_{i}}+c(x, u)-\rho\right]=0$, where $\phi: \mathbb{R}^{d} \rightarrow \mathbb{R}$ and $\rho$ is a scalar. Under certain conditions we establish the existence of a unique viscosity solution $(\phi, \rho)$ of the above equation. Finally we characterize the optimal control via this unique solution.

The existence of an optimal control for this problem has been studied in [3], [4], [6], [10]. In these works the existence of an optimal control has been established under suitable conditions. However, one does not have the existence of an optimal control for arbitrary initial law. Under 
a certain Liapunov type stability condition we have got rid of this limitation. To the best of our knowledge the viscosity solution of the HJB equation for the ergodic control problem has not been studied thus far.

Our paper is organized as follows. Section 2 deals with notation and preliminaries. In Section 3 we establish the existence of an optimal control. The HJB equation is studied in Section 4.

\section{NOTATION AND PRELIMINARIES}

Let $V$ be a compart metric space and $U=\mathcal{P}(V)$ the space of probability measures on $V$ endowed with the topology of weak convergence. We consider the $d$-dimensional controlled diffusion process $X(\cdot)=\left[X_{1}(\cdot), \ldots, X_{d}(\cdot)\right]^{\prime}$ described by

$$
\begin{aligned}
d X(t) & =\int_{V} b(X(t), y) u(t)(d y) d t+\sigma(X(t)) d W(t) \\
X(0) & =X_{0}
\end{aligned}
$$

where $b(\cdot, \cdot)=\left[b_{1}(\cdot, \cdot), \ldots, b_{d}(\cdot, \cdot)\right]^{\prime}: \mathbb{R}^{d} \times V \rightarrow \mathbb{R}^{d}$ is the drift vector, $\sigma(\cdot)=\left[\sigma_{i j}(\cdot)\right]: \mathbb{R}^{d} \rightarrow \mathbb{R}^{d \times d}$ is the diffusion matrix, $X_{0}$ is an $\mathbb{R}^{d}$-valued random variable with a prescribed law, $W(\cdot)=\left[W_{1}(\cdot), \ldots W_{d}(\cdot)\right]^{\prime}$ is a $d$-dimensional standard Wiener process independent of $X_{0}, u(\cdot)$ is a $U$-valued process with measurable sample path satisfying the following nonanticipativity property : for $t \geq s, W(t)-W(s)$ is independent of $\{u(r), W(r), r \leq s\}$. Such a process is called an admissible relaxed control. It is called an admissible precise control if $u(\cdot)=\delta_{v(\cdot)}$ for a $V$-valued process $v(\cdot)$ satisfying the same nonanticipativity condition. We make the following assumptions. We denote various constants by $C_{i}$.

(A1) (i) $b$ is continuous and for $x, y \in \mathbb{R}^{d}$

$$
\sup _{v \in V}|b(x, v)-b(y, v)| \leq C_{1}|x-y|
$$


(ii) $\sigma$ is continuous, and for $x, y \in \mathbb{R}^{d}$

$$
|\sigma(x)-\sigma(y)| \leq C_{2}|x-y|
$$

Under (A1), for a prescribed admissible control the equation (2.1) has a unique strong solution. For $v \in V, f \in W_{\text {loc }}^{2, p}\left(\mathbb{R}^{d}\right), x \in \mathbb{R}^{d}$, let

$$
L^{v} f(x)=\frac{1}{2} \sum_{i, j=1}^{d} a_{i j}(x) \frac{\partial^{2} f(x)}{\partial x_{i} \partial x_{j}}+\sum_{i=1}^{d} b_{i}(x, v) \frac{\partial f(x)}{\partial x_{i}}
$$

where $a_{i j}(\cdot)=\sum_{k=1}^{d} \sigma_{i k}(\cdot) \sigma_{j k}(\cdot)$, and for $u \in U$

$$
L^{u} f(x)=\int_{V} L^{v} f(x) u(d v) .
$$

An admissible control (relaxed or precise) $u(\cdot)$ is called feedback if $u(\cdot)$ is progressively measurable with respect to the natural filtration of $X(\cdot)$. In such a case (2.1) will not admit a strong solution in general. We say that $(X(\cdot), u(\cdot))$ is a stationary relaxed solution of the controlled martingale problem for $L$ if $(X(\cdot), u(\cdot))$ is a stationary process such that $u(\cdot)$ is a relaxed feedback control and $X(\cdot)$ the corresponding controlled diffusion.

We now state the following result which plays a very crucial role in the existence of an optimal control. For a proof see [9].

Theorem 2.1. If $\mu \in \mathcal{P}\left(\mathbb{R}^{d} \times V\right)$ satisfies

$$
\int L^{v} f(x) \mu(d x, d v)=0 \text { for all } f \in \mathcal{D}\left(\mathbb{R}^{d}\right)
$$

then there exists a stationary relaxed solution $(X(\cdot), u(\cdot))$ of the controlled martingale problem for $L$ such that for each $t \geq 0$

$$
E\left[\int_{V} f(X(t), v) u(t)(d v)\right]=\int_{\mathbf{R}^{d} \times V} f d \mu, \quad \text { for all } f \in C_{b}\left(\mathbb{R}^{d} \times V\right) .
$$


Let $c: \mathbb{R}^{d} \times V \rightarrow \mathbb{R}$ be the cost function. We make the following assumption on $c$.

(A2) $c$ is continuous and for $x, y \in \mathbb{R}^{d}$

$$
\sup _{v \in V}|c(x, v)-c(y, v)| \leq C_{3}|x-y| .
$$

Our aim is to minimize the long run average (or ergodic) cost

$$
\limsup _{T \rightarrow \infty} \frac{1}{T} E \int_{0}^{T} \int_{V} c(X(t), v) u(t)(d v) d t
$$

over all admissible relaxed controls.

We will carry our program under the following 'stability' assumption.

(A3) For $x, y \in \mathbb{R}^{d}, v \in V$, let

$$
\begin{aligned}
a(x) & =\sigma(x) \sigma^{\prime}(x), \sigma(x, y)=\sigma(x)-\sigma(y) \\
a(x, y) & =\sigma(x, y) \sigma^{\prime}(x, y) .
\end{aligned}
$$

We assume that there exist a symmetric positive definite matrix $Q$ and a constant $\alpha>0$ such that for $x, y \in \mathbb{R}^{d}, v \in V$,

$$
\begin{aligned}
2(b(x, v)-b(y, v))^{\prime}(Q(x-y)) & -\frac{(Q(x-y))^{\prime} a(x, y)(Q(x-y))}{(x-y)^{\prime} Q(x-y)} \\
& +\operatorname{tr}(a(x, y) Q) \leq-\alpha|x-y|^{2}
\end{aligned}
$$

The following example will show the assumptions (A1) and (A3) arise naturally.

Example. Let $V=[0,1]^{d}, b(x, v)=B x+D v, \sigma_{1}(x)=x$ and $\sigma_{i}(x)=0$ for all $i \neq 1$. Here $B, D$ are constant $d \times d$ matrices where all eigenvalues of $B$ have negative real part and $\sigma_{j}$ is the $j$ th column of $\sigma$. It is easy to see that $(A 1)$ is satisfied. Now notice that, there exist a positive definite matrix $Q$ such that $B^{\prime} Q+Q B=-I$. Using this, one obatins (A3): for $x \neq y$,

$$
2(b(x, v)-b(y, v))^{\prime}(Q(x-y))-\frac{(Q(x-y))^{\prime} a(x, y)(Q(x-y))}{(x-y)^{\prime} Q(x-y)}
$$




$$
\begin{aligned}
& +\operatorname{tr}(a(x, y) Q) \\
& =(x-y)^{\prime}\left(B^{\prime} Q+Q B\right)(x-y)-\frac{\left((x-y)^{\prime} Q(x-y)\right)^{2}}{(x-y)^{\prime} Q(x-y)} \\
& +\operatorname{tr}\left((x-y)(x-y)^{\prime} Q\right) \\
& =-|x-y|^{2}-(x-y)^{\prime} Q(x-y)+(x-y)^{\prime} Q(x-y) \\
& =-|x-y|^{2} .
\end{aligned}
$$

We will now establish the 'asymptotic flatness of the flow' under (A1), (A3). We closely follow the arguments in [2].

Lemma 2.1. Let $u(\cdot)$ be any admissible relaxed control. Let $X(x, t)$ be the corresponding solution with initial condition $X(0)=x$. Then under (A1), (A3) there exist constants $C_{4}>0, C_{5}>0$ independent of $u(\cdot)$ such that for any $x, y \in \mathbb{R}^{d}$

$$
E|X(x, t)-X(y, t)| \leq C_{4} e^{-C_{5} t}|x-y| .
$$

Proof. Consider the Liapunov function

$$
w(x)=\left(x^{\prime} Q x\right)^{1 / 2} .
$$

The function $w$ may be modified near the origin to make it a $C^{2}$ function on all of $\mathbb{R}^{d}$.

Write $\partial_{k}$ to denote the partial differentiation with respect to the $k$-th coordinate and let

$$
\tilde{L}^{v}=\sum_{i=1}^{d}\left(b_{i}(x, v)-b_{i}(y, v)\right) \partial_{i}+\frac{1}{2} \sum_{i, j=1}^{d} a_{i j}(x, y) \partial_{i} \partial_{j} .
$$

Then using (A3), for $x \neq y$,

$$
\tilde{L}^{v} w(x-y) \leq-\frac{\gamma}{2}\{(x-y) Q(x-y)\}^{-1 / 2}|x-y|^{2},
$$

for some $\gamma>0$. Hence for some constant $C_{4}>0$

$$
\tilde{L}^{v} w(x-y) \leq-C_{4} w(x-y) .
$$


Let $\tau=\inf \{t \geq 0 \mid X(x, t)=X(y, t)\}$ (possibly $+\infty)$. Now by Ito's formula,

$$
\begin{aligned}
E w(X(x, t \wedge \tau) & -X(y, t \wedge \tau))-w(x-y) \\
& =E \int_{0}^{t \wedge \tau} \int_{V} \tilde{L}^{v} w(X(x, s)-X(y, s)) u(s)(d v) d s \\
& \leq-C_{4} E \int_{0}^{t \wedge \tau} w(X(x, s)-X(y, s)) d s .
\end{aligned}
$$

For $t \geq \tau, X(x, t)=X(y, t)$ a.s. by the pathwise uniqueness of the solution of (2.1). Thus by Gronwall's inequality it follows that for any $t \geq 0$

$$
E w(X(x, t)-X(y, t)) \leq w(x-y) e^{-C_{4} t},
$$

Finally using the positive definiteness of $Q,(2.5)$ follows from (2.8).

The arguments in [ Proposition 3.1,1] can be mimicked to get the following result.

Lemma 2.2. Let $u(\cdot)$ be any admissible relaxed control and $X(x, t)$ the corresponding process with initial condition $X(0)=x$. Then under (A1) and (A3) there exist a constant $\delta>0$ and a function $g(\cdot)$ such that for any $t>0$,

$$
E|X(x, t)|^{1+\delta}<g(x)
$$

\section{EXISTENCE RESULTS}

In this section we establish the existence of an optimal control under (A1), (A2), (A3). Our arguments closely follow those in [4, Chapter 6], therefore we present here the bare sketches to illustrate the main ideas.

Let

$$
\Gamma=\left\{\mu \in \mathcal{P}\left(\mathbb{R}^{d} \times V\right) \mid \int_{\mathbb{R}^{d} \times V} L^{v} f d \mu=0 \quad \forall f \in \mathcal{D}\left(\mathbb{R}^{d}\right)\right\} .
$$


For $\mu \in \Gamma$, let

$$
\rho_{\mu}=\int c d \mu
$$

Using (A2), Theorem 2.1 and Lemma 2.2, it can be shown that

$$
\sup _{\mu \in \Gamma} \int|c|^{1+\delta} d \mu \quad<\infty
$$

where $\delta>0$ is as in Lemma 2.2. Let

$$
\rho^{\star}=\inf _{\mu \in \Gamma} \rho_{\mu} .
$$

Lemma 3.1. The set $\Gamma$ in (3.1) is compact.

Proof. In view of $[4$, Lemma 5.1] it suffices to show that there exists a $C^{2}$ Liapunov function

$$
w: \mathbb{R}^{d} \rightarrow \mathbb{R}
$$

such that

$$
\lim _{|x| \rightarrow \infty} L^{v} w(x) \rightarrow-\infty \text { uniformly in } v
$$

Now consider the Liapunov function

$$
w(x)=\left(x^{\prime} Q x\right)^{1 / 2}
$$

with a suitable modification near the origin already considered in the proof of Lemma 2.1. This clearly satisfies (3.5). Hence the result follows from [4, Lemma 5.1].

Corollary 3.1. There exists a $\mu^{*} \in \Gamma$ such that

$$
\rho^{\star}=\int c d \mu^{\star}
$$

Proof. From (3.3) it follows that $\mu \mapsto \int c d \mu$ is continuous on $\Gamma$. Since $\Gamma$ is compact the result follows.

Let $\left(X^{\star}(\cdot), u^{\star}(\cdot)\right)$ be the stationary relaxed solution of the controlled martingale problem corresponding to $\mu^{\star}$. Then if the law of $\left(X^{\star}(0), u^{\star}(0)\right)$ is such that 


$$
E\left[\int_{V} f\left(X^{\star}(0), v\right) u^{\star}(0)(d v)\right]=\int_{\mathbf{R}^{d} \times V} f d \mu^{\star} \forall f \in C_{b}\left(\mathbb{R}^{d} \times V\right),
$$

then

$$
\lim _{T \rightarrow \infty} \frac{1}{T} E \int_{0}^{T} \int_{V} c\left(X^{\star}(s), v\right) u^{\star}(s)(d v) d s=\int c d \mu^{\star}=\rho^{\star} .
$$

Now fix the probability space and the Wiener process $W(\cdot)$ on which $u^{\star}(\cdot)$ is defined. Consider the corresponding process $X^{\star}(\cdot)$ with varying initial law. Using (2.5) we can argue as in [Theorem 1.2.2, 4] that (3.6) holds for any initial law of $X^{\star}(0)$. We can then mimick the arguments in $[4$, Chapter 6$]$ to conclude the following.

Theorem 3.1. Under any admissible relaxed control $u(\cdot)$ and any initial law, the corresponding solution $X(\cdot)$ of $(2.1)$ satisfies

$$
\liminf _{T \rightarrow \infty} \frac{1}{T} E \int_{0}^{T} \int_{V} c(X(s), v) u(s)(d v) d s \geq \rho^{\star} .
$$

Thus $u^{\star}(\cdot)$ as above is optimal.

Remark 3.1. Note that (3.7) establishes a much stronger optimality of $u^{\star}(\cdot)$, viz., the most "pessimistic" average cost under $u^{\star}(\cdot)$ is no worse than the most "optimistic" average cost under any other admissible control.

\section{HAMILTON-JACOBI-BELLMAN EQUATION}

The HJB equation for the ergodic control problem is

$$
\inf _{u \in U}\left[L^{u} \phi(x)+\bar{c}(x, u)-\rho\right]=0
$$

where,

$$
\bar{c}(x, u)=\int_{V} c(x, v) u(d v),
$$

$\phi: \mathbb{R}^{d} \rightarrow \mathbb{R}$ is a suitable function and $\rho$ is a scalar. Solving the HJB equation means finding a suitable pair $(\phi, \rho)$ satisfying $(4.1)$ in an appropriate sense. For the nondegenerate case under certain assumptions 
one can find a unique solution $(\phi, \rho)$ of $(4.1)$ in a certain class such that $\phi$ is $C^{2}[4$, Chapter 6$]$. To obtain analogous results for the degenerate case we introduce the notion of a viscosity solution of (4.1) ([7], [8]). Let $\phi \in C\left(\mathbb{R}^{d}\right)$ and $\rho \in \mathbb{R}$.

Definition 4.1. The pair $(\phi, \rho)$ is said to be a viscosity solution of $(4.1)$ if for any $\psi \in C^{2}\left(\mathbb{R}^{d}\right)$

$$
\inf _{u \in U}\left[L^{u} \psi(x)+\bar{c}(x, u)-\rho\right] \geq 0
$$

at each local maximum $x$ of $(\phi-\psi)$, and

$$
\inf _{u \in U}\left[L^{u} \psi(x)+\bar{c}(x, u)-\rho\right] \leq 0
$$

at each local minimum $x$ of $(\phi-\psi)$.

Let

$$
G=\left\{f: \mathbb{R}^{d} \rightarrow \mathbb{R} \mid f(0)=0, f \text { is Lipschitz continuous }\right\} .
$$

We will show that (4.1) has a unique viscosity solution in $G \times \mathbb{R}$. To this end we follow the traditional vanishing discount method. Let $\lambda>0$. For an admissible relaxed control $u(\cdot)$, let

$$
\phi_{\lambda}^{u}(x)=E\left[\int_{0}^{\infty} e^{-\lambda t} \bar{c}(X(t), u(t)) d t \mid X(0)=x\right] .
$$

Let $\phi_{\lambda}$ denote the discounted value function, i.e.

$$
\phi_{\lambda}(x)=\inf _{u(\cdot)} \phi_{\lambda}^{u}(x)
$$

Let

$$
\begin{aligned}
H=\left\{f: \mathbb{R}^{d} \rightarrow \mathbb{R} \quad \mid\right. & f \text { is continuous and }|f(x)| \leq C(1+|x|) \\
& \text { for some constant } C\} .
\end{aligned}
$$

By the results of [8] (see also [7]) $\phi_{\lambda}$ is the unique viscosity solution in $H$ of the following $\mathrm{HJB}$ equation for the discounted control problem 


$$
\inf _{u}\left[L^{u} \phi_{\lambda}(x)+\bar{c}(x, u)-\lambda \phi_{\lambda}(x)\right]=0 .
$$

Theorem 4.1. Under (A1), (A2) and (A3), (4.1) has a viscosity solution in $G \times \mathbb{R}$.

Proof. For $x, y \in \mathbb{R}^{d}$ we have

$$
\begin{aligned}
& \left|\phi_{\lambda}(x)-\phi_{\lambda}(y)\right| \\
\leq & \sup _{u(\cdot)} E\left[\int_{0}^{\infty}\left(e^{-\lambda t}|\vec{c}(X(x, t), u(t))-\vec{c}(X(y, t), u(t))|\right) d t\right] \\
\leq & C_{3} \sup _{u(\cdot)} \int_{0}^{\infty} e^{-\lambda t} E|X(x, t)-X(y, t)| d t \\
\leq & C_{3} C_{4} \int_{0}^{\infty} e^{-\lambda t} e^{-C_{5} t}|x-y| d t=\frac{C_{3} C_{4}}{\lambda+C_{5}}|x-y| .
\end{aligned}
$$

Thus there exists a constant $C_{6}$ independent of $\lambda$ such that

$$
\left|\phi_{\lambda}(x)-\phi_{\lambda}(y)\right| \leq C_{6}|x-y|
$$

Let $\tilde{\phi}_{\lambda}(x)=\phi_{\lambda}(x)-\phi_{\lambda}(0)$. Then $\tilde{\phi}_{\lambda}$ is the unique viscosity solution in $H$ to

$$
\inf _{u}\left[L^{u} \tilde{\phi}_{\lambda}(x)+\bar{c}(x, u)-\lambda \tilde{\phi}_{\lambda}(x)+\lambda \phi_{\lambda}(0)\right]=0
$$

Let $\left\{\lambda_{n}\right\}$ be a sequence such that $\lambda_{n} \rightarrow 0$ as $n \rightarrow \infty$. From (4.10) it follows using Ascoli's theorem that $\tilde{\phi}_{\lambda_{n}}$ converges to a function $\phi \in C\left(\mathbb{R}^{d}\right)$ uniformly on compact subsets of $\mathbb{R}^{d}$ along a suitable subsequence of $\left\{\lambda_{n}\right\}$. By $(2.9), \lambda \phi_{\lambda}(0)$ is bounded in $\lambda$. Hence along a suitable subsequence of $\left\{\lambda_{n}\right\}$ (still denoted by $\left\{\lambda_{n}\right\}$ by an abuse of notation) $\lambda_{n} \phi_{\lambda_{n}}(0)$ converges to a scalar $\rho$ as $n \rightarrow \infty$. Thus by the stability property of viscosity solution ([7], [8]) it follows that the pair $(\phi, \rho)$ is a viscosity solution of (4.1). Clearly $\phi(0)=0$ and from (4.10) it follows that $\phi$ is Lipschitz continuous. Thus $(\phi, \rho) \in G \times \mathbb{R}$.

Theorem 4.2. Under (A1), (A2), (A3), if $(\phi, \rho) \in G \times \mathbb{R}$ is a viscosity solution of (4.1) then $\rho=\rho^{\star}$. 
Proof. Let $\gamma>0$. Since $(\phi, \rho)$ is a viscosity solution of $(4.1)$, therefore $\phi$ is a viscosity solution of

$$
\inf _{u}\left[\left(L^{u}-\gamma\right) \phi(x)+\bar{c}(x, y)-\rho+\gamma \phi(x)\right]=0 .
$$

Therefore by the uniqueness of viscosity solution ([7], [8]), it can be shown as in [8] that

$$
\phi(x)=\inf _{u} E\left[\int_{0}^{\infty} e^{-\gamma t}\{\bar{c}(X(t), u(t))-\rho+\gamma \phi(X(t))\} d t\right] .
$$

Let $\mu \in \Gamma$. Let $(X(\cdot), u(\cdot))$ be the stationary solution of the martingale problem corresponding to $\mu$. Then fix the probability space and the Wiener process $W(\cdot)$ on which $u(\cdot)$ is defined and consider the process $X(\cdot)$ with varying initial condition. Then for any $x \in \mathbb{R}^{d}$

$$
\gamma \phi(x)+\rho \leq \gamma E\left[\int_{0}^{\infty} e^{-\gamma t} \bar{c}(X(t), u(t)) d t\right]+\gamma^{2} e\left[\int_{0}^{\infty} e^{-\gamma t} \phi(X(t)) d t\right] .
$$

Letting $\gamma \rightarrow 0$, and arguing as before using (2.5), we have

$$
\rho \leq \int \bar{c} d \mu
$$

Hence $\rho \leq \rho^{\star}$. To get the reverse inequality let $u^{\gamma}(\cdot)$ be a an optimal relaxed feedback control for the cost criterion in (4.13). Let $\mathcal{F}_{t}=\sigma(X(s), s \leq t)$. Then

$$
e^{-\gamma t} \phi(X(t))+\int_{0}^{t} e^{-\gamma s}\left\{\bar{c}\left(X(t), u^{\gamma}(t)\right)-\rho+\gamma \phi(X(t))\right\} d t
$$

is an $\mathcal{F}_{t}$-martingale ([4]). By Lemma 2.2 it is uniformly integrable. Therefore letting $\gamma \rightarrow 0$, we can argue as in [Lemma 1.1,5] to show that for some relaxed feedback control $\bar{u}(\cdot)$

$$
\phi(X(t))+\int_{0}^{t}(\bar{c}(X(t), \bar{u}(t))-\rho) d t
$$

is an $\mathcal{F}_{t}$-martingale. Taking expectation, dividing by $t$ and letting $t \rightarrow$ $\infty$, we obtain 


$$
\rho \geq \liminf _{t \rightarrow \infty} \frac{1}{t} E \int_{0}^{t} \bar{c}(X(s), \bar{u}(s)) d t \geq \rho^{\star} .
$$

Theorem 4.3. Under (A1), (A2) and (A3), if $\left(\phi, \rho^{\star}\right) \in G \times \mathbb{R}$ is a viscosity solution of (4.1). Then

(i) If $u$ is any admissible relaxed control then $\phi(X(t))+\int_{0}^{t}(\bar{c}(X(s)$, $\left.u(s))-\rho^{\star}\right) d s$ is an $\mathcal{F}_{t}$-submartingale, where $\mathcal{F}_{t}=\sigma(X(s), s \leq t)$

(ii) If for some admissible relaxed control $u(\cdot)$

$$
\phi(X(t))+\int_{0}^{t}\left(\bar{c}(X(s), u(s))-\rho^{\star}\right) d s
$$

is an $\mathcal{F}_{t}$-martingale then $u$ is optimal.

(iii) If $u$ is an admissible relaxed control which is optimal and the process $(X(\cdot), u(\cdot))$ is stationary with $E\left[\int_{V} f(X(t), v) u(t)(d v)\right]=$ $\int_{\mathbf{R}^{d} \times V} f d \mu, m u \in \mathcal{P}\left(\mathbb{R}^{d} \times V\right)$ for each $t$, then

$$
\phi(X(t))+\int_{0}^{t}\left(\bar{c}\left(X(s), u(s)-\rho^{\star}\right) d s\right.
$$

is an $\mathcal{F}_{t}$-martingale.

\section{Proof.}

(i) Let $u(\cdot)$ be any admissible relaxed control. By (4.12)

$$
e^{-\gamma t} \phi(X(t))+\int_{0}^{t} e^{-\gamma s}\left\{\bar{c}(X(s), u(s))-\rho^{\star}+\gamma \phi(X(s))\right\} d s
$$

is an $\mathcal{F}_{t}$-submartingale [4]. Letting $\gamma \rightarrow 0$, (i) follows.

(ii) Since

$$
\phi(X(t))+\int_{0}^{t}\left(\bar{c}\left(X(s), u(s)-\rho^{\star}\right) d s\right.
$$

is an $\mathcal{F}_{t}$-martingale under $u(\cdot)$, we have

$$
E \phi(X(t))+E \int_{0}^{t}\left(\bar{c}(X(s), u(s))-\rho^{\star}\right) d t=\phi(x)
$$

by taking $X(0)=x$. Therefore 


$$
\frac{1}{t} E \int_{0}^{t} \bar{c}(X(s), u(s)) d s=\rho^{\star}+\frac{1}{t} E \phi(X(t))+\frac{\phi(x)}{t} .
$$

Letting $t \rightarrow \infty$ and using Lemma 2.2

$$
\lim _{t \rightarrow \infty} \frac{1}{t} E \int_{0}^{t} \bar{c}(X(s), u(s)) d s=\rho^{\star} .
$$

Thus $u(\cdot)$ is optimal.

(iii) Let

$$
M_{t}=\phi(X(t))+\int_{0}^{t}\left(\bar{c}(X(s), u(s))-\rho^{\star}\right) d s .
$$

Then $E M_{t}=\int \phi d \mu$. Suppose $M_{t}$ is not a martingale. Then there exists $t>s>0$ and $A \in \mathcal{F}_{s}$ with $P(A)>0$ such that

$$
E\left[M_{t} \mid \mathcal{F}_{s}\right]>\quad M_{s} \text { on } A
$$

Now,

$$
\begin{aligned}
\int \phi d \mu= & E M_{t}=E\left[E\left[M_{t} \mid \mathcal{F}_{s}\right]\right. \\
= & E\left[E\left[M_{t} I_{A} \mid \mathcal{F}_{s}\right]\right] \\
& +E\left[E\left[M_{t} I_{A^{c}} \mid \mathcal{F}_{s}\right]\right] \\
= & E\left[I_{A} E\left[M_{t} \mid \mathcal{F}_{s}\right]\right]+E\left[I_{A^{c}} E\left[M_{t} \mid \mathcal{F}_{s}\right]\right] \\
> & E M_{s}=\int \phi d \mu
\end{aligned}
$$

which is a contradiction.

Theorem 4.4. The equation (4.1) has a unique viscosity solution in $G \times \mathbb{R}$.

Proof. Let $(\phi, \rho) \in G \times \mathbb{R}$ and $\left(\psi, \rho^{\prime}\right) \in G \times \mathbb{R}$ be two viscosity solutions of (4.1). Then by Theorem $4.2 \rho=\rho^{\prime}=\rho^{\star}$. Let $u^{\star}(\cdot)$ be an optimal relaxed feedback control such that the process $(x(\cdot), u(\cdot))$ is stationary and for each $t$

$$
E\left[\int_{V} f(X(t), v) u^{\star}(t)(d v)\right]=\int_{\mathbf{R}^{d} \times V} f d \mu \quad \forall \quad f \in C_{b}\left(\mathbb{R}^{d} \times V\right) .
$$


Then by Theorem 4.3

$$
\phi(X(t))+\int_{0}^{t}\left(\bar{c}\left(X(s), u^{\star}(s)\right)-\rho^{\star}\right) d s
$$

and

$$
\psi(X(t))+\int_{0}^{t}\left(\bar{c}\left(X(s), u^{\star}(s)\right)-\rho^{\star}\right) d s
$$

are $\mathcal{F}_{t}$-martingales and therefore so is $\phi(X(t))-\psi(X(t))$. By Lemma 2.2 it is uniformly integrable and therefore converges a.s. Hence $\phi-\psi$ is a constant $c^{\prime}$ on the support $M$ of $\bar{\mu}$, where $\bar{\mu}$ is the marginal of $\mu$ on $\mathbf{R}^{d}$. Without loss of generality assume that $c^{\prime} \geq 0$ (otherwise consider $\psi-\phi)$. If $0 \in M$ then $c^{\prime}=0$. If $0 \notin M$, assume $c^{\prime}>0$. Then for some $\varepsilon>0, \phi-\psi>\frac{c^{\prime}}{2}$ on an $\varepsilon$-neighbourhood $M_{\varepsilon}$ of $M$ and $0 \notin M_{\varepsilon}$. Let

$$
\eta=\inf \left\{t>0 \mid X(t) \in \partial M_{\varepsilon}\right\}
$$

We claim that $E \eta<\infty$ for any $x \notin M_{\varepsilon}$. Indeed, let $x \notin M_{\varepsilon}$ and $y \in M$.

Then since $M$ is an invariant set for $X(\cdot)$ under $u^{\star}(\cdot)$

$$
\begin{aligned}
P(\eta>t) & \leq P(|X(x, t)-X(y, t)|>\varepsilon) \\
& \leq \frac{C_{4}}{\varepsilon} e^{-C_{5} t}|x-y|
\end{aligned}
$$

by (2.5) and Chebyshev's inequality. Thus

$$
E \eta \leq \frac{C_{4}}{\varepsilon C_{5}}|x-y| \text {. }
$$

We can mimick the arguments in [Proposition 3.1, 1] to show that for some $\delta>0$

$$
E|X(\eta)|^{1+\delta}<\infty
$$

In view of $(4.15 \mathrm{a})$ and $(4.15 \mathrm{~b})$ the optimal control problems of minimizing

$$
E\left[\int_{0}^{\eta}\left(\bar{c}(X(t), u(t))-\rho^{\star}\right) d t+\phi(X(\eta))\right]
$$

and 


$$
E\left[\int_{0}^{\eta}\left(\bar{c}(X(t), u(t))-\rho^{\star}\right) d t+\psi(X(\eta))\right]
$$

over all admissible relaxed controls are well posed. Optimal relaxed controls for these problems can be shown to exist [4]. Let $u(\cdot)$ be an optimal relaxed feedback control for (4.16). Then

$$
\phi(X(\eta \wedge t))+\int_{0}^{\eta \wedge t}\left(\bar{c}(X(s), u(s))-\rho^{\star}\right) d s
$$

is a martingale and

$$
\psi(X(\eta \wedge t))+\int_{0}^{\eta \wedge t}\left(\bar{c}(X(s), u(s))-\rho^{\star}\right) d s
$$

is a submartingale. Thus $\psi(X(\eta \wedge t))-\phi(X(\eta \wedge t))$ is a submartingale.

Therefore

$$
E \psi(X(\eta \wedge t))-E \phi(X(\eta \wedge t)) \geq \psi(x)-\phi(x)
$$

Taking $x=0$ and letting $t \rightarrow \infty$, we get $c^{\prime}<0$, a contradiction. Thus $c^{\prime}=0$. By the same argument we have $\psi(x)-\phi(x) \leq 0$ for any $x \in \mathbb{R}^{d}$. Similary we can show that for any $x \in \mathbb{R}^{d}$

$$
\phi(x)-\psi(x) \leq 0
$$

Hence $\phi \equiv \psi$.

\section{REFERENCES}

[1] G.K. Basak, A class of limit theorems for singular diffusions, J. Multivariate Analysis 39 (1991), 44-59.

[2] G.K. Basak and R.N. Bhattacharya, Stability in distribution for a class of singular diffusions, Annals of Probability 20 (1992), $312-321$.

[3] A.G. Bhatt and V.S. Borkar, Occupation measures for controlled Markov processes: Characterization and optimality, Preprint.

[4] V.S. Borkar, Optimal control of diffusion processes, Pitman Research notes in Maths No. 203, Longman Scientific and Technical, Harlow, 1989 
[5] V.S. Borkar, On extremal solutions to stochastic control problem, Appl. Math. Optim. 24 (1991), 317-330.

[6] V.S. Borkar, A note on ergodic control of degenerate diffusions, Preprint.

[7] W.H. Fleming and H.M. Soner, Controlled Markov processes and viscosity solutions, Springer-Verlag, New York, 1991.

[8] P.L. Lions, Optimal control of diffusion processes and HamiltonJacobi-Bellman equations, Part II: viscosity solutions and uniqueness. Communications on P.D.E. 8(1993), 1229-76.

[9] R.H. Stockbridge, Time-average control of a martingale problem. Existence of a stationary solution, Annals of Probability 18 (1990), 190-205.

[10] R.H. Stockbridge, Time-average control of a martingale problems : a linear programming formulation, Annals of Probability 18 (1990), 206-217. 